\section{MORTE SÚBITA NA EPILEPSIA: TODOS OS CAMINHOS LEVAM AO CORAÇÃO}

A relação entre freqüência cardíaca $(\mathrm{FC})$ e expectativa de vida, tão bem avaliada pelo professor Luiz Antonio Machado Césarr ${ }^{1}$, nos estimulou essa correspondência aos editores.

A epilepsia é a doença neurológica crônica grave mais comum. Nos países desenvolvidos, a incidência da epilepsia é de aproxi madamente 50 por 100.000 indivíduos/ano, sendo mais freqüente entre crianças e idosos $\mathrm{S}^{2,3}$. $0 \mathrm{~s}$ fatores etiológicos mais comuns são os tumores encefálicos, traumatismo crânio-encefálico, acidente vascular encefálico e infecções do sistema nervoso central ${ }^{2}$. Nos países em desenvolvimento, infecções endêmicas (neurocisticercose e malária) apresentam-se como principais fatores de risco². Curiosamente, indivíduos com epilepsia apresentam duas ou três vezes mais chances de morrerem subitamente quando comparados com indivíduos sem epilepsia, sendo que a categoria de morte mais comum nesses casos é o fenômeno da morte súbita e inesperada na epilepsia (SUDEP) ${ }^{3}$.

Na SUDEP, o óbito deve ocorrer de maneira não-traumática, sem afogamento, pode ter ou não relatos de crise, excetuando-se status epilepticus, e os exames realizados após a morte não podem revelar causas anatômicas ou toxicológicas para a morte ${ }^{4}$. A incidência da SUDEP é considerada alta em pacientes com epilepsia crônica (1-2) 1,000 pessoas/ano) e maior nos indivíduos com refratários ao tratamento farmacológico (3-9/1,000 pessoas/ano) ${ }^{5}$. Apesar desses dados epidemiológicos, alguns fatores podem ser considerados de risco para a SUDEP: idade, início precoce das epilepsias, tempo de duração das epilepsias, não controle e freqüência das crises epilépticas, tipos de crises epilépticas, regime de drogas antiepilépticas adotado e temperaturas frias ${ }^{5,6}$.

Apesar do mecanismo patofisiológico da SU DEP ser desconhecido, uma possível explicação é que este seja de origem cardiogênica. N esse sentido, estudos funcionais do coração têm sido realizados com 0 intuito de desvendar o fenômeno da SUDEP. Diversos estudos têm investigado possíveis alterações do sistema cardiovascular durante os períodos ictal e interictal de pacientes com epilepsia. Durante as crises epilépticas, a avaliação concomitante do EEG/ECG tem demonstrado que as crises epilépticas estão diretamente asso ciadas com um aumento da freqüência cardíaca. Marshal et al. ${ }^{7}$ avaliaram 12 pacientes que foram monitorados por meio de video-EEG e ECG, simultaneamente, observando a ocorrência de taquicardia durante as crises epilépticas. Paralelamente, Zijlmans et al. ${ }^{8}$ observaram que ocorre um aumento na freqüência cardíaca de pelo menos 10 batimentos por minuto imediatamente antes do início das crises em $93 \%$ dos pacientes e em $23 \%$ das crises ( $49 \%$ dos pacientes), a taquicardia precedeu o início clínico e eletrográfico das crises.

Além disso, Tigaran et al. ${ }^{9}$ demonstraram que $40 \%$ dos pacientes com epilepsia apresentaram depressão do segmento ST durante ou logo após a crise epiléptica e um outro estudo demonstrou uma lentificação do prolongamento QT simultaneamente ao aparecimento de alterações eletrográficas detectadas no EEG, sendo que essas alterações cardíacas foram mais pronunciadas nos pacientes que morreram subitamente quando comparados com os que não evoluíram para óbito ${ }^{10}$.

Por outro lado, é interessante frisarmos que alguns dados clínicos sugerem 0 envolvimento do sistema nervoso parassimpático durante as crises epilépticas, evidenciado por bradicardia sinusal, a qual pode ser consequêencia da apnéia registrada durante as crises ${ }^{11}$. Além das alterações verificadas durante as crises epilépticas, alguns estudos têm relatado anormalidades funcionais do sistema cardiovascular em pacientes com epilepsia durante o período interictal. No final da década de 80 , avaliou-se concomitantemente 0 EEG/ECG de 338 pacientes com epilepsia com o intuito de evidenciar a presença de arritmias. Verificouse que $5 \%$ dos pacientes apresentavam alterações do ritmo, embora essa taxa de anormalidade não difira daquelas encontradas em adultos saudáveis e assintomáticos ${ }^{12}$.

Em 1993, Drake et al. ${ }^{13}$ verificaram um aumento da freqüência cardíaca e da duração do prolongamento QT de 75 pacientes com epilepsia quando submetidos ao EC G. N 0 entanto, estas alterações não estavam fora dos padrões de normalidade. Além disso, os autores relataram que os pacientes com crises parciais complexas e crises secundariamente generalizadas apresentam uma maior freqüência ventricular e duração do intervalo QT quando comparados com indivíduos com epilepsia generalizada ${ }^{13}$.

Recentemente, pesquisadores dinamarqueses utilizaram testes cardiológicos (ECG, monitorização por Holter, ecocardiografia, teste ergométrico e cintilografia miocárdica) com o intuito de avaliar possíveis alterações cardiovasculares em 23 pacientes com epilepsia refratária9. Dessa forma, 40\% dos pacientes avaliados apresentaram depressão do segmento ST, o qual foi associado com o aumento da frequêencia cardíaca durante as crises epilépticas. Sendo assim, os autores concluíram que um processo de isquemia cardíaca pode ocorrer nos pacientes com epilepsia e que são refratários ao tratamento farmacológico ${ }^{9}$. Do ponto de vista experimental, nosso grupo de pesquisa foi pioneiro em avaliar a FC in vivo (ECG) e in vitro (preparação de Langendorf) de ratos com epilepsia' ${ }^{14}$. N ossos resultados mostraram diferenças significantes na FC in vivo entre os grupos estudados (animais do grupo controle: $307 \pm 9$ bpm; animais com epilepsia: $346 \pm 7 b p m)$. Em contraste, não encontramos diferenças entre os grupos nos experimentos in vitro. Dessa forma, concluímos que sob a influência do sistema nervoso central, o coração pode apresentar alterações funcionais que aumentam a probabilidade de ocorrência de morte súbita nas epilepsias.

Finalmente, gostaríamos de parabenizar o professor Luiz Antonio Machado César pelo excelente artigo de revisão e o próximo passo para nós, epileptologistas, será entender e associar os mecanismos pelos quais a FC pode influenciar o sistema cardiovascular de pacientes com epilepsia. 0 entendimento desses mecanismos será importante para o desenvolvimento de novas estratégias na prevenção da SUDEP.

Fulvio Alexandre Scorza Ricardo Mario Arida Marly de Albuquerque Esper Abräo Cavalheiro São Paulo - SP 
Referências

1. César LAM. Freqüência cardíaca e risco cardiovascular. Rev Assoc Med Bras. 2007:53:456-9.

2. Duncan JS, Sander JW, Sisodiya SM, Walker MC. Adult epilepsy. Lancet. 2006;367:1087-100

3. Sander JW. The epidemiology of epilepsy revisited. Curr $\mathrm{O}$ pin $\mathrm{N}$ eurol. 2003;16:165-70.

4. $N$ ashef L. Sudden unexpected death in epilepsy: terminology and definitions. Epilepsia. 1997;38(Suppl 4):S6-S8.

5. Stollberger $C$, Finsterer J. Cardiorespiratory findings in sudden unexplained/ unexpected death in epilepsy (SU D EP). Epilepsy Res. 2004;59:51-60.

6. Scorza FA, de Albuquerque M, Arida RM, C avalheiro EA. Sudden unexpected death in epilepsy: are winter temperatures a new potential risk factor? Epilepsy Behav. 2007;10:509-10.

7. Marshall DW, W estmoreland BF, Sharbrough FW. Ictal tachycardia during temporal lobe seizures. Mayo Clin Proc. 1983;58:443-46.

8. Zijlmans M, Flanagan D, Gotman J. Heart rate changes and ECG abnormalities durring epileptic seizures: prevalence and definition of an objective clinical sign. Epilepsia. 2002;43:847-54.
9. Tigaran MD, M olgaard H, M cclealland R, D am M, Jaffe AS. Evidence of cardiac ischemia during seizures in drug refractory epilepsy patients. N eurology. 2003;60:492-95.

10. Tavernor SJ, Brown SW, Tavernor RM, G ifford C. Electrocardiograph Q T lengthening associated with epileptiform EEG discharges: a role in sudden unexplained death in epilepsy? Seizure. 1996;5:79-83.

11. N ashef L, W alker F, Allen P, Sander JW, Shorvon SD, Fish D R. Apnoea and bradycardia during epileptic seizures: relation to sudden death in epilepsy. J N eurol N eurosurg Psychiatry. 1996;60:297-300.

12. Keilson MJ, Hauser WA, Magrill JP, Goldman M. EcG abnormalities in patients with epilepsy. N eurology. 1987;37:1624-6.

13. Drake MF, Raider $C R$, Kay A. Electrocardiography in epilepsy patients without cardiac symptoms. Seizure. 1993;2:63-5.

14. Colugnati DB, Gomes PA, Arida RM, Albuquerque M, Cysneiros RM, $C$ avalheiro $E A$, et al. Analysis of cardiac parameters in animals with epilepsy: possible cause of sudden death? Arq Neuropsiquiatr. 2005;63:1035-41. 